\title{
Gas Concentration Measurement System Based on DSP and Ultrasonic Technology
}

\author{
Xue Wang ${ }^{1, a}$, Yi Jin ${ }^{2, b}$, Jianhui Wei $^{3, c}$, Dingyun Jin $^{4, d}$ \\ ${ }^{1,2,3,4}$ changchun engineering technology college, Changchun, 130117, China \\ aemail: lylglg@163.com, bemail:kxu20112012@163.com
}

Keywords: DSP; Ultrasound; Gas Concentration; FFT; Autocorrelation Algorithm; Weak Signal

\begin{abstract}
On the basis of full analysis of gas detection instruments, the use of DSP and ultrasonic technology to design a new type of gas concentration detection system. DSP is the core of the whole system, mainly complete the DDS chip sequence control, special A/D sampling control and external FLAS $\mathrm{H}$ and SDRAM memory chip control, and to communicate with PC through serial port. Discussed the principle of ultrasound detection of gas concentration, as well as within the DSP to realize time domain signal autocorrelation operation, to improve the signal-to-noise ratio. Hardware structure diagram and software design of the system are given with the block diagram, the experiment proved that the system well meet the requirements of the gas concentration detection, can be used in a variety of gas detection.
\end{abstract}

\section{Introduction}

Gas detection instruments application field widely, covering the industrial and agricultural production, environmental quality monitoring, national defense, aerospace, family life and other fields. Infrared gas detection instrument is one of today's mainstream gas detector. Infrared gas detection technology is a kind of direct contact of the real-time monitoring of gas detection technology, with strong selectivity, stable performance, high sensitivity, large dynamic range, the interference of equipment and the advantages of long service life. For inflammable and explosive gas, carbon dioxide gas monitoring, has the irreplaceable advantages, has been more and more widely attention and application.

Common optical gas detection technology include Non-Dispersive Infrared, NDIR, spectrophotometric measurement, TDLS, PAS technology, etc. Among them, the wide band, the simplest non-dispersed Infrared gas sensor structure, stable performance, quick, high precision, suitable for light and handheld devices, has the huge commercial value [1]. In 2002, the British Heriot Watt - university of JG Crowder and others by optimizing device optical structure, make into the super lens surface detector, thereby reducing the area of the detector and increased the radiation detectors receiving area. The experimental results show that the concentration of NO2 gas at room temperature detecting resolution of 2 PPM. In 2009, the university of Barcelona, Spain J.F onollosa r\&d testing such as ethylene $(\mathrm{C} 2 \mathrm{H} 4)$ gas concentration device applied to the fruit of the stored procedure, the instrument, the minimum detectable concentration of ethylene to 30 PPM. Russia loffe physics institute of technology In S.A leksandrov et using LED and Se In photoelectric diode of "light on" for methane gas detection, the minimum detectable methane concentration to 170 PPM. These research results in the lab has been greatly exceeded industrial detection accuracy requirement of standard. They all are adopted by the dispersive infrared (NDIR) gas concentration detection technology. Visible, the domestic study of infrared gas detection device with improved there is still a huge improvement space. Infrared gas detection device in the process of design, use, in order to ensure the accuracy of measurement, should be considered for regularly or irregularly calibration instrument and calibration, to make up for measuring error caused by temperature change, the need for instrument for temperature compensation. In this paper, we study a kind of simple and effective based on DSP and the infrared spectrum absorption technology of gas concentration detection method, on the one hand, can improve the infrared gas detection instrument in the research, development, production efficiency; On the other hand the measure precision of the 
instrument can be improved and better temperature compensation.

\section{Detection Principle}

\section{The principle of ultrasound detection.}

Gas concentration in the ultrasonic testing method can be divided into two kinds of single channel detection and multi-channel detection, the two methods in the channel, the basic principle of the acoustic pulse transmitting and receiving are all the same [2].

Ultrasonic testing technique is suitable for the gas concentration of binary gas mixtures, and even multiple trace detection of a single gas composition in the mixed gases, [3]. For binary gas, under the experimental conditions of atmospheric pressure, can use the ideal gas model to analyze it.

Set the quality of the gas a and b constant pressure heat capacity of Cpa and Cpb respectively, the quality of constant volume heat capacity of Cva and Cvb respectively, the relative molecular mass of Mra and Mrb respectively, the binary mixture of average quality constant pressure constant

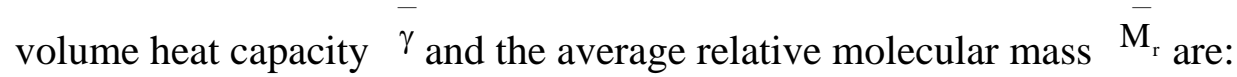

$$
\begin{aligned}
& \bar{\gamma}=\frac{n C_{p a}+(1-n) C_{p b}}{n C_{v a}+(1-n) C_{v b}} \\
& \bar{M}_{r}=n M_{r a}+(1-n) M_{r a}
\end{aligned}
$$

Type $\mathrm{n}$ for gas in a concentration in the mixture. And average velocities of mixed gas is:

$$
\bar{c}^{2}=\frac{\bar{\gamma} R T}{\overline{M_{r}}}
$$

$\mathrm{T}$ for the thermodynamic temperature of the gas type, for gas constant $\mathrm{R}$, if that

$$
Y=\frac{\bar{c}^{2}}{R T}=\frac{\bar{\gamma}_{\bar{r}}}{\bar{M}_{r}}
$$

Can be measured by ultrasonic pulse propagation in the fixed acoustic distance $\mathrm{L}$ time $\mathrm{t}$, the sound velocity obtained by $\bar{c}=L / t$, plug type on the value of $Y$.

By above all tidy:

$$
A n^{2}+B n+C=0
$$

Because $^{0 \leq n \leq 1}$, this type can solution of concentration for gas a:

$$
n=\frac{-B+\sqrt{B^{2}-4 A C}}{2 A}
$$

Type A, B and C are A function of Y, and

$$
\begin{aligned}
& A=M_{r a} C_{v a}+M_{r b} C_{v b}-M_{r a} C_{v b}-M_{r b} C_{v b} \\
& B=\left(M_{r a} C_{v b}+M_{r b} C_{v a}-2 C_{v b} M_{r b}\right) Y-C_{p a}+C_{p b} \\
& C=M_{r a} C_{v b} Y-C_{p a}
\end{aligned}
$$

DSP autocorrelation detection principle.

A measured signal $x(k)$ by the useful signal s $(k)$ and noise of $u(k)$ :

$\mathrm{x}(\mathrm{k})=\mathrm{s}(\mathrm{k})+\mathrm{u}(\mathrm{k})$

$\mathrm{X}(\mathrm{k})$ of autocorrelation function is:

$R_{\mathrm{x}}(m)=R_{\mathrm{s}}(m)+R_{\mathrm{su}}(m)+R_{\mathrm{us}}(m)+R_{\mathrm{u}}(m)$ 
$R_{\mathrm{s}}(m)$ is the autocorrelation function of $\mathrm{s}(\mathrm{k}) . R_{\mathrm{su}}(m)$ is the cross-correlation function of $\mathrm{s}(\mathrm{k})$ and $\mathrm{u}(\mathrm{k}) .{ }_{\mathrm{us}}(\mathrm{m})$ is the cross-correlation function of $\mathrm{u}(\mathrm{k})$ 与 $\mathrm{s}(\mathrm{k}) .{ }_{\mathrm{u}}(\mathrm{m})$ is the autocorrelation function of $\mathrm{u}(\mathrm{k})$. Because the circuit is mainly for the white noise in the system, $R_{\mathrm{su}}(m)=R_{\mathrm{us}}(m)=0$, With the increase of $\mathrm{m}, R_{\mathrm{u}}(m) \rightarrow 0, R_{\mathrm{s}}(m)=\lim _{N \rightarrow \infty} \frac{1}{2 N+1} \sum_{k=-N}^{N} x(k) x(k+m)$.

According to wiener - king James principle, power signal autocorrelation function and power spectral density function with Fourier transform relationship, so you can use FFT to calculate the autocorrelation function. However, the actual sampling value only the $\mathrm{N}+1$ point, so $R_{\mathrm{x}}(m)$ find out only estimates of the autocorrelation function. When using FFT to calculate the autocorrelation function, $x(k)$ must fill $\mathrm{N}$ zero, make the length of the $2 \mathrm{~N}+1$, so the power spectral density as follows:

$$
S_{\mathrm{x}}(m)=S_{\mathrm{s}}(\omega)=\frac{1}{2 N+1} \sum_{m=-N}^{N} R_{s}(m) e^{-j \omega m}=\frac{1}{2 N+1}\left|X_{2 N+1}\left(e^{-j \omega m}\right)\right|^{2}
$$

Can get to IFFT on type $R_{s}(m)$.

Gas concentration measurement system hardware block diagram is shown in figure 1. It mainly includes the sensor of excitation signal and the selection of sensor location, signal acquisition and the design of the external memory four parts. It is the core of ADSP - BF531 chip, which is responsible for the whole system coordination and implementation algorithm function. The working process of the system, the DSP chip by setting SPI controller, control of DDS chip D9850 to produce a particular stable amplitude of ultrasonic frequency sinusoidal signal. Through the signal amplifier and multi-channel switch, exert on the specified drive electrode sensor, and drive with frequency vibration sensor. Sensor output signal via the preamplifier amplification to the appropriate level, and then after A high precision A/D chip AD9235 sampling will transform the results sent to DSP related operations, to calculate the vibration amplitude.

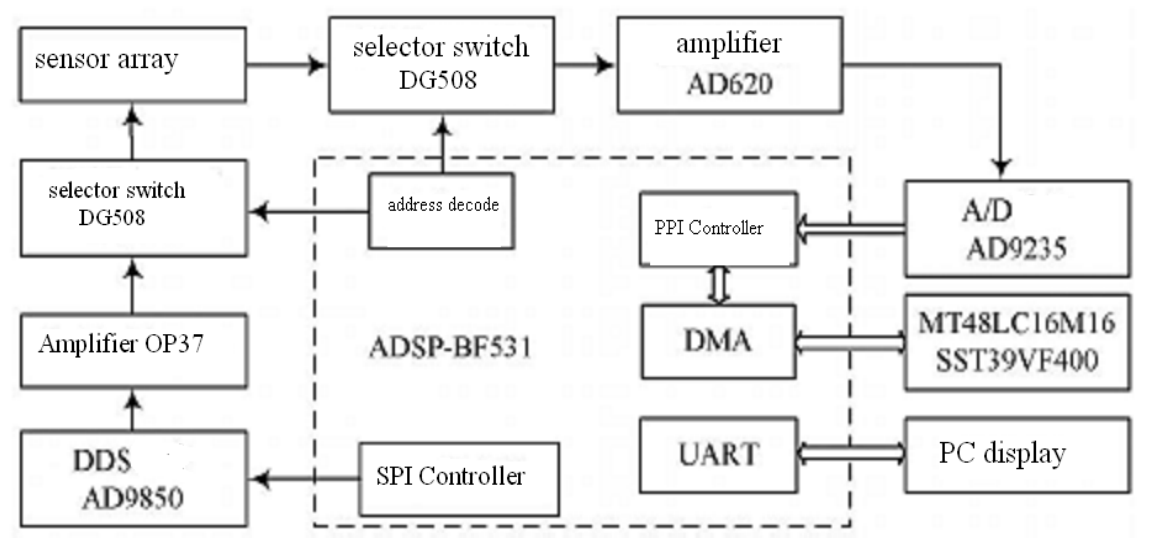

Fig.1 The hardware block diagram of the system

After the completion of the frequency sweep, DSP to searching spectral peak frequency response curve, determine the resonant frequency of the sensor, so as to determine the corresponding concentrations of the gas sensor. And multi-channel switch information can determine the sensor position, eventually get a different location information of gas concentration.

\section{Software Design of the System}

Gas concentration detection system software design including the DSP initialization program, the interrupt to scale, applications, including response to external interrupt program, data post-processing. Detection system is started the first running internal ROM curing Boo $\mathrm{t}$ - Loader program, will be stored in the FLASH program code loaded into the DSP internal RAM. Program is first initialized system, through the internal registers configuration DSP, DSP specific working condition is determined, and then the interrupt response program and data post-processing. System 
program block diagram as shown in figure 2, the related calculation done in the interrupt subprogram, all the procedures for the convenience of transplantation is completed using structured C language.

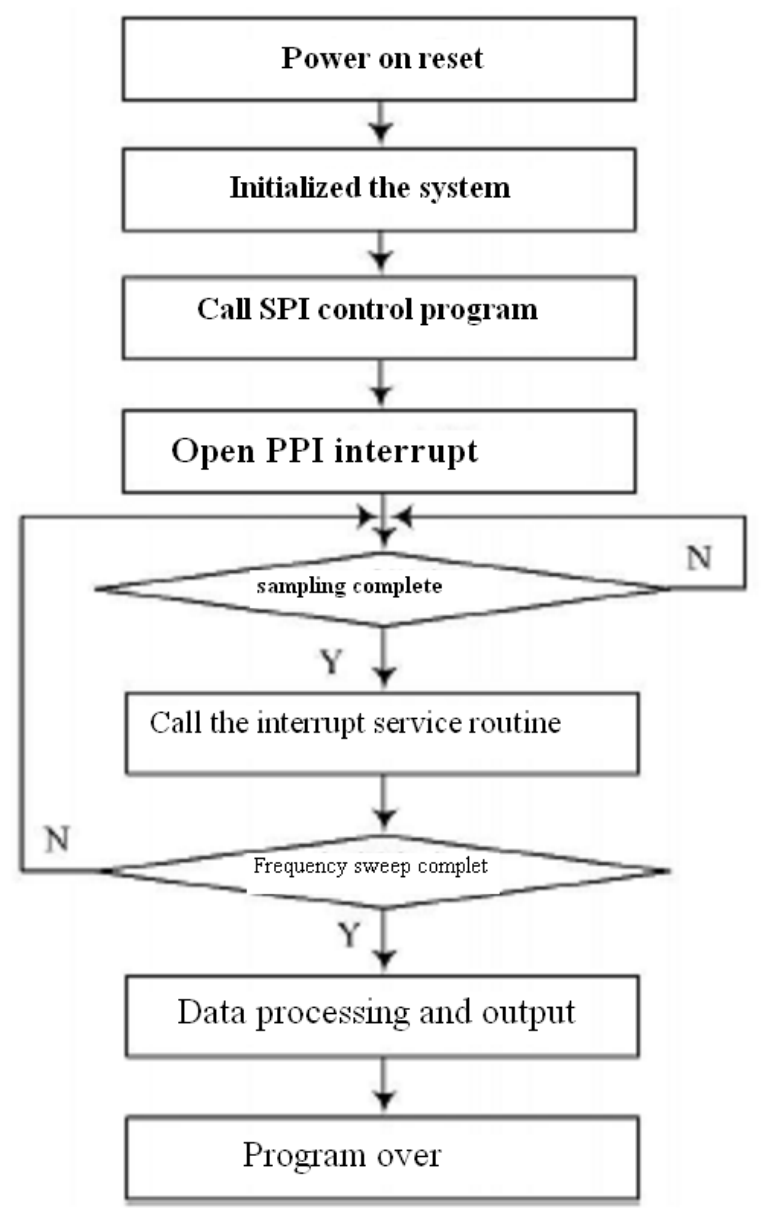

Fig. 2 program block diagram of the system

\section{Conclusion}

Experiments prove that gas concentration measurement system based on DSP and ultrasonic technology overcomes the shortcomings inherent in traditional gas concentration detecting instrument, can effectively detect the weak signal of the transducer. System with Blackfin531 as the core, comprehensive utilization of the DSP technology, autocorrelation detection method and ultrasonic technology, the hardware design is simple and reliable, using structured C language program, good versatility and portability, easy to DSP implementation. System well meets the need of gas concentration detection, high accuracy of measurement, method is simple and can be used in the detection of hydrogen and other gases.

\section{References}

[1] Dell'la M, Cannizo M , Diritti A. Measurement of high-pressure natural gas flow using ultrasonic flowmelers [J]. Measurement, 1997, 20(2): 75-89.

[2] HansV , PoppenG, Vonlavante E, et al.Vortex shedding flowmeters and ultrasound deeetion: signal proeessing and influence of bluff bodygeometry[J]. Flow Measurement and Instrumentation, 1998, 9(2): 79-82.

[3] Castaings M, Hosten B. The use of electrostatic, ultrasonic, aircoupled transducers to generate and receive lamb waves in anisotropic viscoelastic plates [J].Ultrasonics, 1998, 36: 361-365. 
[4] Worch A. Clamp-on ultrasonic cross-correlation flowmeter for one phase flow [J]. Measurement Science and Technology, 1998, 9 (4): 622-630.

[5] Haoqiang Tan. C language programming[M].Beijing: Tsinghua university press,2013:46-52. 